\title{
Experimental Observation of the Aubry Transition in Two-Dimensional Colloidal Monolayers
}

\author{
T. Brazda, ${ }^{1}$ A. Silva, ${ }^{2}$ N. Manini, ${ }^{2}$ A. Vanossi, ${ }^{3,4}$ R. Guerra, ${ }^{2,5}$ E. Tosatti, ${ }^{4,6,3}$ and C. Bechinger ${ }^{1,7, *}$ \\ 12. Physikalisches Institut, Universität Stuttgart, Pfaffenwaldring 57, 70569 Stuttgart, Germany \\ ${ }^{2}$ Dipartimento di Fisica, Università degli Studi di Milano, Via Celoria 16, 20133 Milano, Italy \\ ${ }^{3}$ CNR-IOM Democritos National Simulation Center, Via Bonomea 265, 34136 Trieste, Italy \\ ${ }^{4}$ International School for Advanced Studies (SISSA), Via Bonomea 265, 34136 Trieste, Italy \\ ${ }^{5}$ Center for Complexity and Biosystems, University of Milan, 20133 Milan, Italy \\ ${ }^{6}$ International Centre for Theoretical Physics (ICTP), Strada Costiera 11, 34151 Trieste, Italy \\ ${ }^{7}$ Fachbereich Physik, Universität Konstanz, Universitätsstrasse 10, 78464 Konstanz, Germany
}

(Received 20 November 2017; revised manuscript received 1 February 2018; published 28 March 2018)

\begin{abstract}
The possibility to achieve entirely frictionless, i.e., superlubric, sliding between solids holds enormous potential for the operation of mechanical devices. At small length scales, where mechanical contacts are well defined, Aubry predicted a transition from a superlubric to a pinned state when the mechanical load is increased. Evidence for this intriguing Aubry transition (AT), which should occur in one dimension (1D) and at zero temperature, was recently obtained in few-atom chains. Here, we experimentally and theoretically demonstrate the occurrence of the AT in an extended two-dimensional (2D) system at room temperature using a colloidal monolayer on an optical lattice. Unlike the continuous nature of the AT in 1D, we observe a first-order transition in 2D leading to a coexistence regime of pinned and unpinned areas. Our data demonstrate that the original concept of Aubry not only survives in 2D but is relevant for the design of nanoscopic machines and devices at ambient temperature.
\end{abstract}

DOI: 10.1103/PhysRevX.8.011050

In the expanding fields of nanoscience, where the competition of length scales is of key importance, Aubry's theoretical concept [1,2], how to achieve frictionless sliding, is one of the most challenging topics in nanotribology [3], with immediate technological applications. The nowadays pervasive tribological concept of superlubricity was originally inferred from the one-dimensional (1D) FrenkelKontorova (FK) class of models [4], describing a chain of interacting particles subject to a periodic substrate potential. Aubry showed that, in the thermodynamic limit and at zero temperature, the 1D incommensurate chain-substrate interface may undergo a second-order transition between an unpinned and a pinned state at a critical value of the substrate corrugation, i.e., contact strength, $U_{0}$. In the unpinned state, the minimum force required to achieve sliding, i.e., the static friction, should vanish: The interface incommensurability can indeed prevent asperity interlocking and collective

\footnotetext{
* Corresponding author. clemens.bechinger@uni-konstanz.de

Published by the American Physical Society under the terms of the Creative Commons Attribution 4.0 International license. Further distribution of this work must maintain attribution to the author(s) and the published article's title, journal citation, and DOI.
}

Subject Areas: Materials Science, Soft Matter, Statistical Physics stick-slip motion of the interface atoms, with a consequent negligibly small frictional force.

After remaining locked away for several decades, Aubrytype signatures were recently observed experimentally in finite 1D linear laser-cooled ion chains on corrugated potentials [5-8]. In contrast, the demonstration and characterization of the Aubry transition in extended and mismatched two-dimensional (2D) monolayers at finite temperature, corresponding to more realistic conditions, is still lacking. Previous experimental work on 2D systems including telescopic dynamics [9] and water flow [10] in carbon nanotubes, sliding of graphite flakes [11-13] and mesas [14-16], graphene nanoribbons [17], cluster nanomanipulation $[18,19]$, or rare-gas island inertial motion [20] has demonstrated superlubricity, i.e., an ultralow static friction, scaling sublinearly with the contact size. However, without the possibility of tuning interaction parameters, the nature of the Aubry transition at 2D interfaces cannot be unraveled and does not disclose how a change from a superlubric to a pinned state occurs.

Here, we report the first microscopically resolved experimental study of the Aubry transition by investigating an extended colloidal layer driven over a laser-generated periodic potential. Unlike conventional substrates, here all relevant physical parameters, such as the lattice periodicity and the contact strength, can be controlled in situ [21]. Unlike $1 \mathrm{D}$, a sliding $2 \mathrm{D}$ crystal will develop a misalignment angle 
relative to the optical lattice, which is crucial for its frictional properties. Our results demonstrate that, around the critical contact strength, the 2D Aubry transition brings about the occurrence of a novel phase separation with a coexistence region between pinned and free-sliding colloidal patches. The frictional rheology of the monolayer is especially interesting and heterogeneous in this two-phase region, which we also characterize by means of molecular-dynamics (MD) simulations mimicking the experimental conditions.

Our experiments were performed at $T_{0} \simeq 298 \mathrm{~K}$, with a suspension of micron-sized polystyrene particles interacting via a repulsive screened Coulomb potential (see Methods section in the Supplemental Material [22]). The colloids form a 2D hexagonal crystal with lattice constant $a_{C}$ at the bottom of the sample cell. A periodic substrate is created by interfering three partial laser beams, as sketched in Fig. 1(a). This optical landscape provides a hexagonal substrate potential, Fig. 1(b), whose amplitude $U_{0}$ can be tuned by the laser intensity. The laser lattice constant $a_{L}$ is set by the intersection angle of the laser beams and allows us to adjust the mismatch ratio $\varepsilon=a_{L} / a_{C}$. Particle positions are tracked by video microscopy with a resolution of about $50 \mathrm{~nm}$ [Fig. 1(b)]. A controlled driving force $F$ of the monolayer is exerted by viscous Stokes forces, which arise when the sample cell is translated horizontally relative to the (resting) interference pattern [22].

Figure 1(c) shows experimental mobilities $\mu$ of an incommensurate $(\varepsilon=0.84)$ colloidal monolayer vs $F$ and for different values of $U_{0}=17-102 k_{B} T_{0}$. For large $U_{0}$, the monolayer is strongly pinned to the substrate and remains immobile until $F$ exceeds the static friction force $F_{s}$, defined by the value where $\mu$ exceeds $10 \%$ of a free sliding (dashed line). Below $U_{0} \leq 38 k_{B} T_{0}$, however, the monolayer becomes mobile already at a minute driving force $F_{\min } \simeq 1 \mathrm{fN}$. This indicates a transition from a pinned to a superlubric state, in agreement with one of the signatures of the Aubry transition. Figure 1(d) shows the static friction force as a function of the corrugation


FIG. 1. Driving a colloidal monolayer across a periodic laser potential. (a) Schematic view of the experimental setup. (b) Corresponding simulated model. (c) Measured mobility of an incommensurate $(\varepsilon=0.84)$ monolayer vs driving force for different corrugation amplitudes $U_{0}$ in the superlubric (red and orange symbols), coexistence (green symbols), and statically pinned phase (blue and black symbols). The dashed line shows the maximum mobility $\left(U_{0}=0\right)$, while the dotted line shows the minimum threshold mobility to detect sliding. The pink arrow points at the crossing of this threshold mobility, defining the value of $F_{s}$ for this value of $U_{0}$. (d) Static friction force $F_{s}$ vs $U_{0}$ obtained from experiments (solid symbols) and simulations (open symbols); the shaded area shows the coexistence region, across which Aubry transition takes place. (e) The monolayer mobility under the action of the smallest experimentally accessible driving force $F_{\min } \simeq 1 \mathrm{fN}$; the critical corrugation $U_{c}=34 k_{B} T_{0}$ is defined by the sharp drop in the mobility (arrow). 
amplitude. To obtain system-independent quantities, $F_{s}$ is normalized to that of a single colloid in the same corrugation potential $F_{1 s}$, and $U_{0}$ to the critical corrugation amplitude $U_{c}$, where the monolayer becomes pinned at $F_{\min }$ [Fig. 1(e)]. In agreement with the predictions by Aubry, our data show the disappearance of static friction below $U_{0} / U_{c} \simeq 1$. Above this transition, $F_{s}$ increases almost linearly and eventually saturates. The open symbols, which were obtained by numerical simulations with parameters adjusted to our experimental conditions [22], show excellent agreement with our data.

Because the Aubry transition is a structural phase transition, it must affect the monolayer microstructure. For stiff monolayers, minimization of the free energy is achieved by rotation of the lattice relative to the substrate by the Novaco angle $\theta_{\text {Nov }}$ [23-24]. Because most particles are slightly displaced from substrate minima after rotation, they become superlubric (unpinned). Contrary to very stiff monolayers, where $\theta_{\text {Nov }}$ has a sharp-defined value that depends on the mismatch ratio (for $\varepsilon=0.84$, one obtains $\theta_{\mathrm{Nov}} \simeq 5^{\circ}$ ) [25], for deformable monolayers, the misfit angle $\theta$ locally varies across the monolayer. Such behavior is confirmed by our experiments as seen by typical particle configurations for different substrate amplitudes [Figs. 2(a)-2(c)]. Red or blue colored particles belong to superlubric domains, which are rotated by $|\theta|>1 / 2 \theta_{\text {Nov }}=2.5^{\circ}$, while grey particles correspond to pinned regions (see Methods section in Ref. [22]). With increasing $U_{0}$, the colloidal lattice becomes increasingly locally aligned with the substrate, as seen by the grey (aligned) domains that proliferate at the expense of
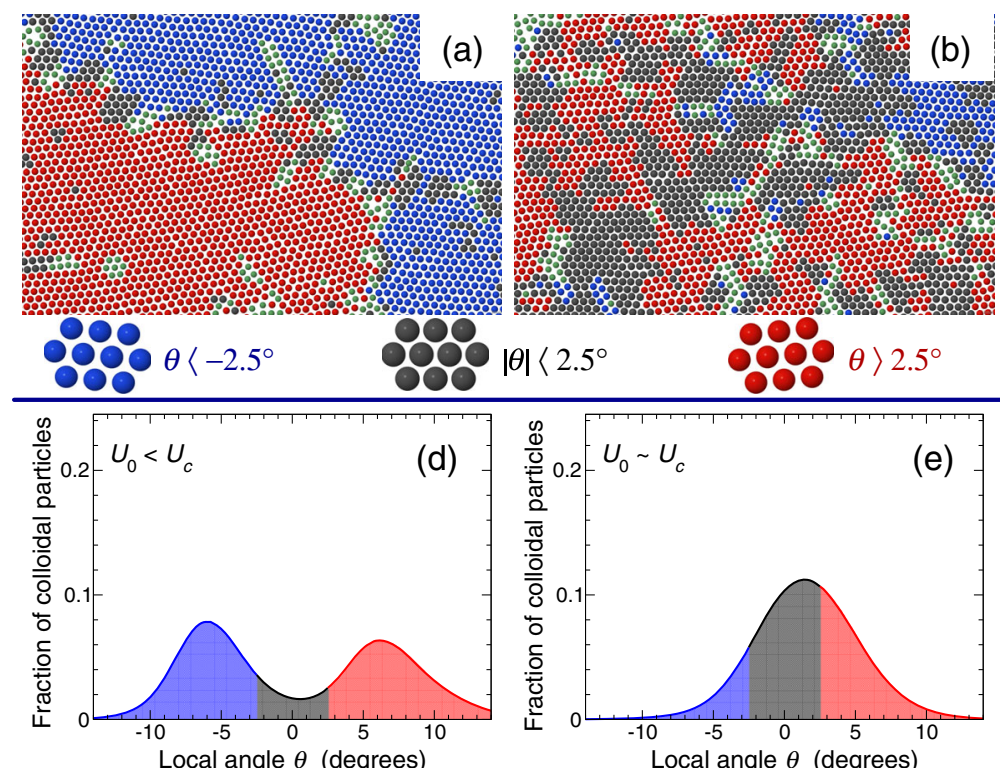

(b)

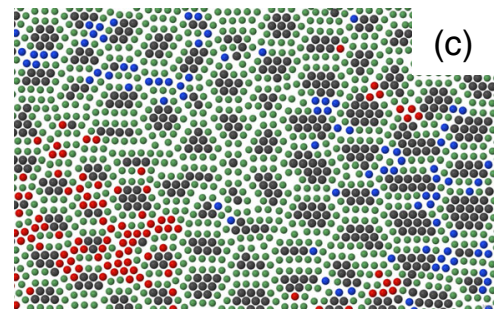

$\theta>2.5^{\circ}$
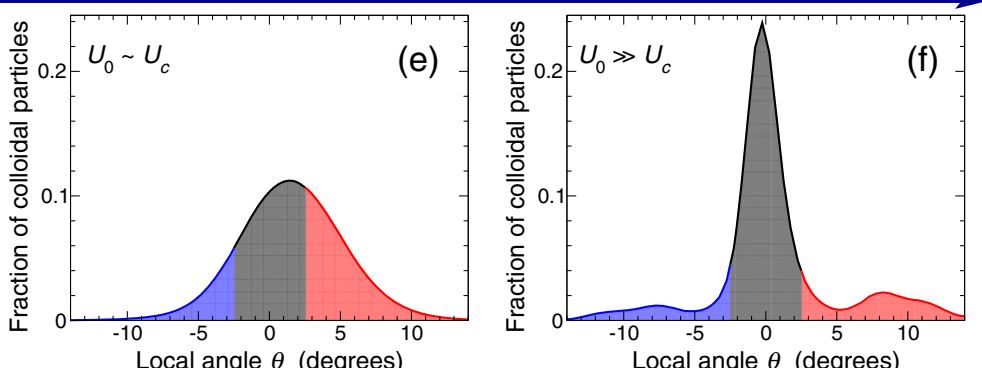

increasing $U_{0}$

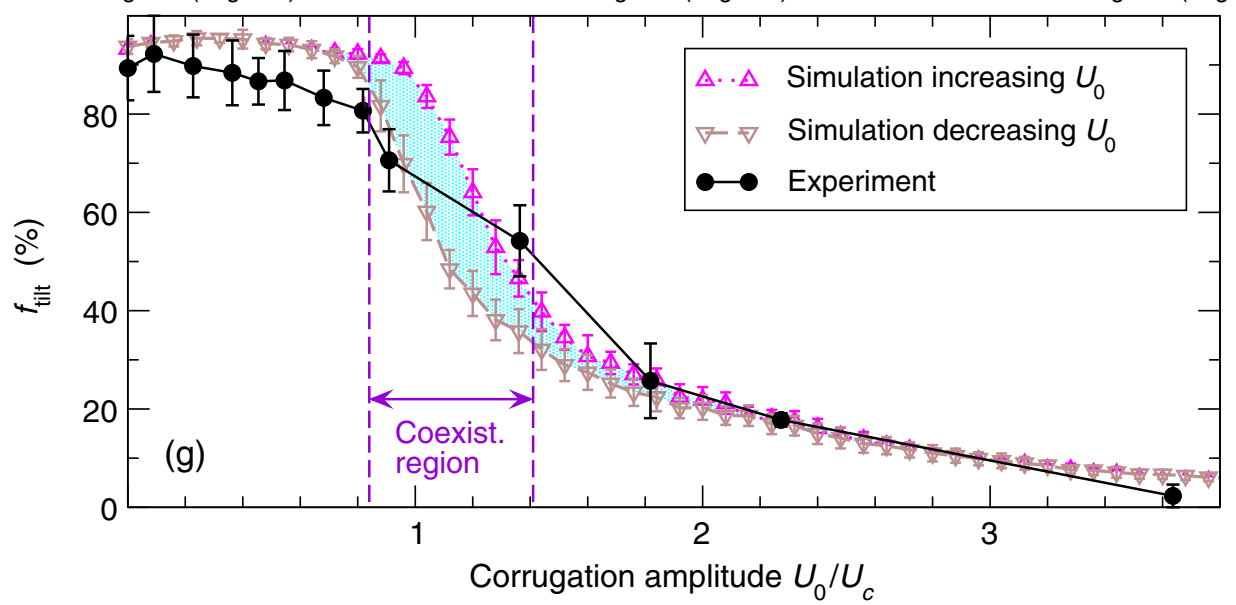

FIG. 2. Structural response and emergence of coexistence region. (a)-(c) Configurations representative of (a) the superlubric phase, (b) the coexistence region, and (c) the pinned phase. The color scheme follows the local bond-orientation angle $\theta_{i}$ relative to the crystalline directions of the corrugation potential (S.M.). (d)-(f) The local-angle distribution, obtained for corrugation (d) $U_{0}=0.63 U_{c}$ (unpinned, i.e. superlubric configuration), (e) $U_{0}=1.25 U_{c}$ (coexistence region), and (f) $U_{0}=11.3 U_{c}$ (statically pinned region). (g) Corrugation dependence of the fraction $f_{\text {tilt }}$ of tilted (red+blue) colloids. In the simulations the presence of hysteresis, due to slow numerical equilibration, helps identify the coexistence region, characterized by a change of slope in experiment. 
tilted regions. For $U_{0}=11.3 U_{c}$ [Fig. 2(c)] essentially only pinned particles are observed. Figures 2(d)-2(f) show how the misfit-angle distribution gradually changes as a function of $U_{0}$. Simulations suggest that the Aubry transition in 2D is of first order, which implies the coexistence of pinned and superlubric regions [26]. The structural features of the three phases are evident in the structure factor analysis reported in Figs. S2 and S3 [22]. Upon ramping $U_{0}$ up and down, simulations exhibit a clear hysteresis in the fraction $f_{\text {tilt }}$ of superlubric particles [Fig. 2(g)]. From the range where error bars show no overlap, the coexistence range between the pinned and unpinned particles can be estimated. Even if, unlike simulations, experimental data are sufficiently well equilibrated and do not show hysteresis, they nonetheless show clear evidence for coexistence [Fig. 2(b)].

Within the 1D FK model, a superlubric-pinned transition is structurally characterized by the displacement of all particles away from the maxima of the corrugation potential [Figs. 3(a)-3(c)]. Following the disorder parameter of
Refs. [27,28], we evaluate the fraction $\Psi$ of colloids at positions where the local substrate potential is above that of the saddle points, namely, the white triangles of Fig. 3(d). This fraction should be $25 \%$ for randomly placed colloids or for $U_{0}=0$, and it decreases with increasing $U_{0}$. This is in agreement with our experimental and simulation data [Figs. 3(e)-3(h)]. It should be emphasized that the $U_{0}$ dependence of $\Psi$ is in good agreement with the corresponding $f_{\text {tilt }}$ behavior in Fig. 2. Both quantifiers support the existence of a first-order Aubry transition at $U_{0} / U_{c} \simeq 1$.

An important novelty in 2D compared to $1 \mathrm{D}$ is that depinning can occur inhomogeneously, nucleating at specific points in the sample. This is especially expected in the phasecoexistence region where, for a given applied force, superlubric patches tilted at the Novaco angle are likely to unpin earlier than nontilted regions. To explore such behavior, we have simulated a configuration near the right end of the coexistence region $\left(U_{0}=1.2 U_{c}\right)$. After applying a weak
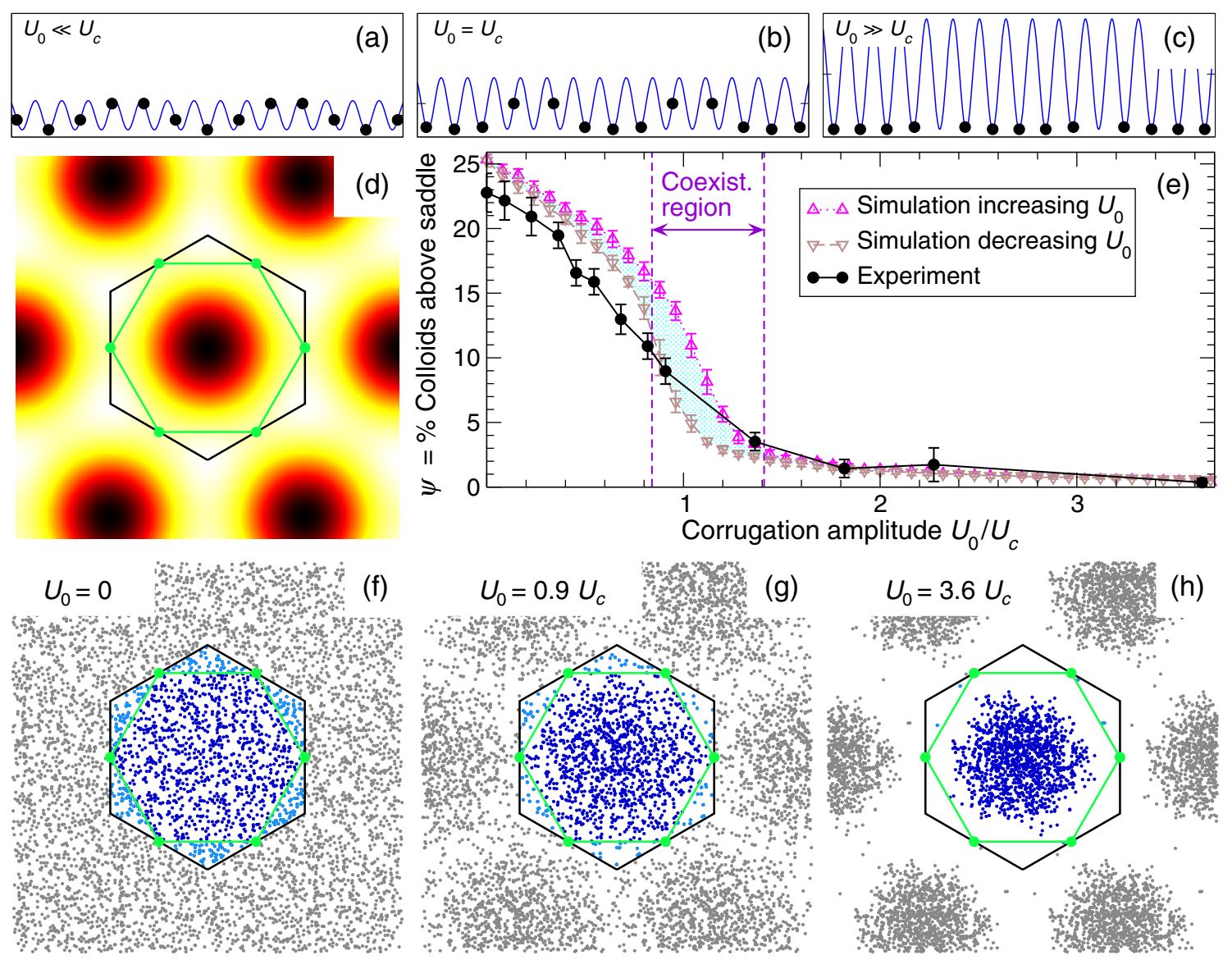

FIG. 3. Local energetics across the Aubry transition. For increasing $U_{0}$, a substrate-mismatched 1D FK chain evolves from a configuration in which (a) minima and maxima regions are populated (superlubric), (b) no particles can be found sitting close to any maximum (pinning threshold), and (c) only regions around the minima are occupied (strongly pinned). (d) The 2D corrugation-potential profile (dark/light $=$ low/high energy); the repulsive regions correspond to the area between the green (saddle-energy contour level) and the black (Wigner-Seitz cell) hexagons. (e) For the 2D colloid crystal, we show the fraction $\Psi$ of colloids in the repulsive regions as a function of $U_{0}$, illustrating the statistical crossover from superlubric to pinned. (f)-(h) Experimental particle positions folded within the Wigner-Seitz cell of the corrugation lattice. 


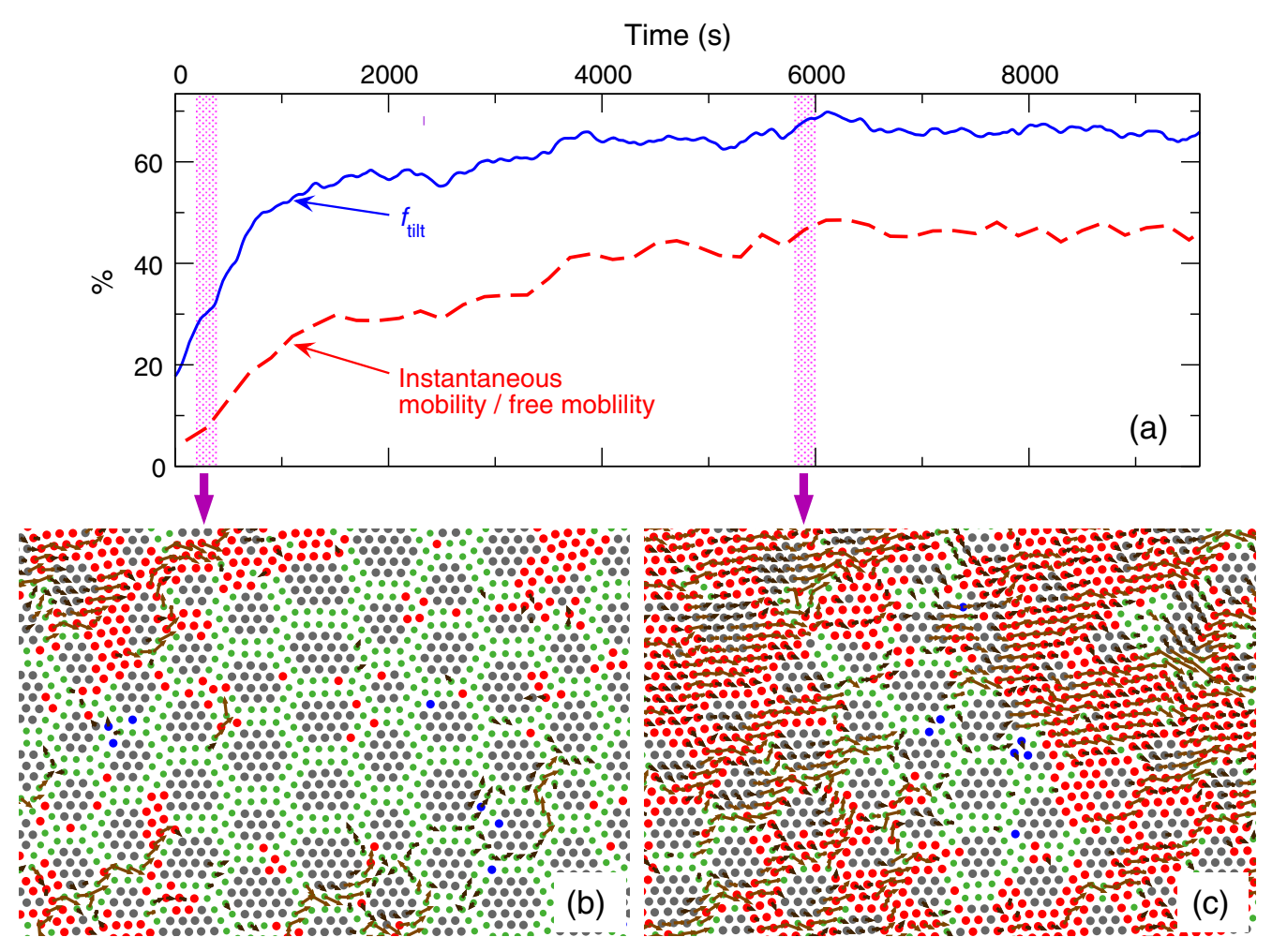

FIG. 4. Force-induced depinning transition. In a simulated pinned configuration near the coexistence region $\left(U_{0}=1.2 U_{c}\right)$, the applied force is raised suddenly from $F=0.73 F_{s}$ to $F=1.09 F_{s}$, at time $t=0$. (a) Both $f_{\text {tilt }}$ and the overall mobility of the colloidal crystal increase steadily in time, until they reach a steady state. Starting from (b) a pinned phase $(t=200 \mathrm{~s})$, the applied force promotes the growth of the (c) superlubric tilted phase $(t=5800 \mathrm{~s})$. The colloid color scheme follows Fig. 2. Brown arrows indicate the displacement patterns over a 200-s time interval. See also the S.M. [22].

force $F<F_{s}, F$ is abruptly increased to $F=1.09 F_{s}$. The red line in Fig. 4(a) reports how the monolayer progressively depins in time. This progressive depinning is understood when examining the simulation movie (S.M.) in Ref. [22], with the local-angle labeling showing a progressive extension of the red and blue unpinned regions. Figures 4(b) and 4(c) report two snapshots of that movie, representative of the mostly pinned initial state and the mostly sliding steady state, respectively. Arrows showing individual particle displacements indicate that initial depinning occurs mainly at the interface regions between the two phases.

Our experimental approach provides a versatile way of studying the conditions under which superlubricity occurs in extended $2 \mathrm{D}$ contacts. In addition, we obtain previously unattainable insights into the microstructural changes occurring during the Aubry transition. For example, the observation of a first-order transition from a superlubric to a pinned state demonstrates that, in contrast to a smooth transition in 1D, as well as in an interesting model of a crystal grain boundary [29], the Aubry transition may remain sharp at finite temperatures for $2 \mathrm{D}$ contacts. The first-order character in our case is boosted by the local angular compliance coupled to the Novaco-McTague rotation. In a different 2D system lacking that compliance, such as a graphene flake on a crystal surface, the first-order character is likely to be weakened. In particular, for micromechanical and nanomechanical devices whose contact areas are rather free from defects, asperities, and long-range elasticity effects [30-32], this should allow for drastic variations in the frictional behavior induced by tiny changes of the mechanical load.

It is a pleasure to acknowledge discussions with Davide Mandelli. Work in Trieste was carried out under ERC Grant No. 320796 MODPHYSFRICT. The COST Action MP1303 is also gratefully acknowledged.

[1] S. Aubry, The New Concept of Transitions by Breaking of Analyticity in a Crystallographic Model, in Solitons, and Condensed Matter Physics, edited by A. R. Bishop and T. Schneider, Springer Series in Solid State Sciences Vol. 8 (Springer, Berlin, 1978), p. 264-277.

[2] M. Peyrard and S. Aubry, Critical Behaviour at the Transition by Breaking of Analyticity in the Discrete Frenkel-Kontorova Model, J. Phys. C 16, 1593 (1983).

[3] A. Vanossi, N. Manini, M. Urbakh, S. Zapperi, and E. Tosatti, Modeling Friction: From Nanoscale to Mesoscale, Rev. Mod. Phys. 85, 529 (2013). 
[4] O. M. Braun and Y. S. Kivshar, Nonlinear Dynamics of the Frenkel-Kontorova Model, Phys. Rep. 306, 1 (1998).

[5] A. Bylinskii, D. Gangloff, I. Counts, and V. Vuletić, Observation of Aubry-type Transition in Finite Atom Chains via Friction, Nat. Mater. 15, 717 (2016).

[6] A. Bylinskii, D. Gangloff, and V. Vuletić, Tuning Friction Atom-by-Atom in an Ion-Crystal Simulator, Science $\mathbf{3 4 8}$, 1115 (2015).

[7] E. Meyer, Controlling Friction Atom by Atom, Science 348, 1089 (2015).

[8] J. Kiethe, R. Nigmatullin, D. Kalincev, T. Schmirander, and T. E. Mehlstäubler, Probing Nanofriction and Aubry-Type Signatures in a Finite Self-Organized System, Nat. Commun. 8, 15364 (2017).

[9] A. Niguès, A. Siria, P. Vincent, P. Poncharal, and L. Bocquet, Ultrahigh Interlayer Friction in Multiwalled Boron Nitride Nanotubes, Nat. Mater. 13, 688 (2014).

[10] E. Secchi, S. Marbach, A. Niguès, D. Stein, A. Siria, and L. Bocquet, Massive Radius-dependent Flow Slippage in Carbon Nanotubes, Nature (London) 537, 210 (2016).

[11] M. Hirano, K. Shinjo, R. Kaneko, and Y. Murata, Observation of Superlubricity by Scanning Tunneling Microscopy, Phys. Rev. Lett. 78, 1448 (1997).

[12] M. Dienwiebel, G. S. Verhoeven, N. Pradeep, J. W. M. Frenken, J. A. Heimberg, and H. W. Zandbergen, Superlubricity of Graphite, Phys. Rev. Lett. 92, 126101 (2004).

[13] X. Feng, S. Kwon, J. Y. Park, and M. Salmeron, Superlubric Sliding of Graphene Nanoflakes on Graphene, ACS Nano 7 , 1718 (2013).

[14] Z. Liu, J. Yang, F. Grey, J. Z. Liu, Y. Liu, Y. Wang, Y. Yang, Y. Cheng, and Q. Zheng, Observation of Microscale Superlubricity in Graphite, Phys. Rev. Lett. 108, 205503 (2012).

[15] E. Koren, E. Lörtscher, C. Rawlings, A. W. Knoll, and U. Duerig, Adhesion and Friction in Mesoscopic Graphite Contacts, Science 348, 679 (2015).

[16] K. M. Liechti, Understanding Friction in Layered Materials, Science 348, 632 (2015).

[17] S. Kawai, A. Benassi, E. Gnecco, H. Söde, R. Pawlak, X. Feng, K. Müllen, D. Passerone, C. A. Pignedoli, P. Ruffieux, R. Fasel, and E. Meyer, Superlubricity of Graphene Nanoribbons on Gold Surfaces, Science 351, 957 (2016).

[18] D. Dietzel, M. Feldmann, U. D. Schwarz, H. Fuchs, and A. Schirmeisen, Scaling Laws of Structural Lubricity, Phys. Rev. Lett. 111, 235502 (2013).
[19] E. Cihan, S. İpek, E. Durgun, and M. Z. Baykara, Structural Lubricity under Ambient Conditions, Nat. Commun. 7, 12055 (2016).

[20] M. Pierno, L. Bruschi, G. Mistura, G. Paolicelli, A. di Bona, S. Valeri, R. Guerra, A. Vanossi, and E. Tosatti, Frictional Transition from Superlubric Islands to Pinned Monolayers, Nat. Nanotechnol. 10, 714 (2015).

[21] T. Bohlein, J. Mikhael, and C. Bechinger, Observation of Kinks and Antikinks in Colloidal Monolayers Driven across Ordered Surfaces, Nat. Mater. 11, 126 (2012).

[22] See Supplemental Material at http://link.aps.org/ supplemental/10.1103/PhysRevX.8.011050 for Methods and a structure-factor analysis. Supplemental Material also includes a movie illustrating the simulated progressive depinning after the driving force is increased abruptly to $F=1.09 F_{s}$.

[23] A. D. Novaco and J. P. McTague, Orientational EpitaxyThe Orientational Ordering of Incommensurate Structures, Phys. Rev. Lett. 38, 1286 (1977).

[24] D. Mandelli, A. Vanossi, N. Manini, and E. Tosatti, Friction Boosted by Equilibrium Misalignment of Incommensurate 2D Colloid Monolayers, Phys. Rev. Lett. 114, 108302 (2015).

[25] F. Grey and J. Bohr, A Symmetry Principle for Epitaxial Rotation, Europhys. Lett. 18, 717 (1992).

[26] D. Mandelli, A. Vanossi, M. Invernizzi, S. Paronuzzi, N. Manini, and E. Tosatti, Superlubric-Pinned Transition in Sliding Incommensurate Colloidal Monolayers, Phys. Rev. B 92, 134306 (2015).

[27] S. N. Coppersmith and D. S. Fisher, Pinning Transition of the Discrete Sine-Gordon Equation, Phys. Rev. B 28, 2566 (1983).

[28] D. Mandelli, A. Vanossi, N. Manini, and E. Tosatti, FiniteTemperature Phase Diagram and Critical Point of the Aubry Pinned-Sliding Transition in a Two-Dimensional Monolayer, Phys. Rev. B 95, 245403 (2017).

[29] F. Lançon, Aubry Transition in a Real Material: Prediction for its Existence in an Incommensurate Gold/Gold Interface, Europhys. Lett. 57, 74 (2002).

[30] M. Cieplak, E. D. Smith, and M. O. Robbins, Molecular Origins of Friction: The Force on Adsorbed Layers, Science 265, 1209 (1994).

[31] G. He, M. H. Müser, and M. O. Robbins, Adsorbed Layers, and the Origin of Static Friction, Science 284, 1650 (1999).

[32] T. A. Sharp, L. Pastewka, and M. O. Robbins, Elasticity Limits Structural Superlubricity in Large Contacts, Phys. Rev. B 93, 121402 (2016). 